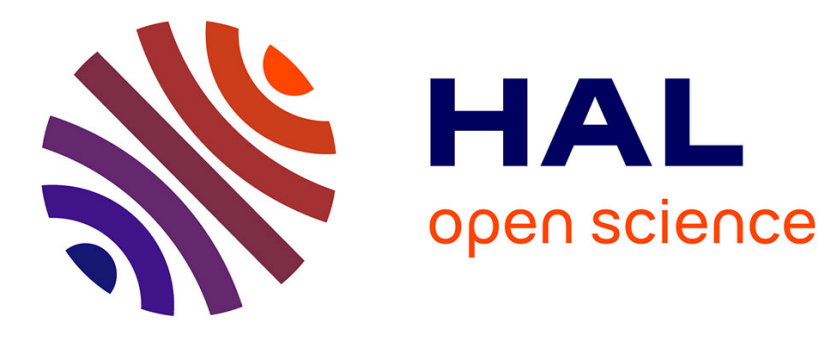

\title{
Wideband Circularly Polarized 3D Wire Antenna for Wi-MAX Applications
}

\author{
Fateh Benmahmoud, Pierre Lemaitre-Auger, Smail Tedjini
}

\section{To cite this version:}

Fateh Benmahmoud, Pierre Lemaitre-Auger, Smail Tedjini. Wideband Circularly Polarized 3D Wire Antenna for Wi-MAX Applications. 2019 IEEE-APS Topical Conference on Antennas and Propagation in Wireless Communications (APWC), Sep 2019, Granada, Spain. pp.250-252, 10.1109/apwc.2019.8870480 . hal-02388108

\section{HAL Id: hal-02388108 https://hal.univ-grenoble-alpes.fr/hal-02388108}

Submitted on 1 Dec 2019

HAL is a multi-disciplinary open access archive for the deposit and dissemination of scientific research documents, whether they are published or not. The documents may come from teaching and research institutions in France or abroad, or from public or private research centers.
L'archive ouverte pluridisciplinaire HAL, est destinée au dépôt et à la diffusion de documents scientifiques de niveau recherche, publiés ou non, émanant des établissements d'enseignement et de recherche français ou étrangers, des laboratoires publics ou privés. 


\section{Wideband Circularly Polarized 3D Wire Antenna for Wi-MAX Applications}

\author{
Fateh Benmahmoud \\ Univ. Grenoble Alpes \\ Grenoble INP, LCIS \\ Valence, France \\ fateh.benmahmoud@1cis.grenoble- \\ inp.fr
}

\author{
Pierre Lemaitre-Auger \\ Univ. Grenoble Alpes \\ Grenoble INP, LCIS \\ Valence, France \\ pierre.lemaitre-auger@1cis.grenoble- \\ inp.fr
}

\author{
Smail Tedjini \\ Univ. Grenoble Alpes \\ Grenoble INP, LCIS \\ Valence, France \\ smail.tedjini@lcis.grenoble-inp.fr
}

\begin{abstract}
In this paper, a crooked wire antenna is optimized to be functional in both the WLAN and the Wi-Max bands (2.4-2.48 and 3.3-3.7 GHz). The antenna presents a right-hand circular polarization (RHCP) with a $3 \mathrm{~dB}$ axial ratio (AR) bandwidth of 3.28 to $4.11 \mathrm{GHz}$. For the WLAN band (2.4-2.48 GHz), the polarization was linear. The antenna is fabricated using 3D printing techniques and occupy a total volume of $33.8 \mathrm{~mm} \times 30.4 \mathrm{~mm} \times 37.4 \mathrm{~mm}$. The simulated and measured farfield results show good agreement with a radiation pattern that assures a near hemispherical coverage toward the upper hemisphere and a measured maximum gain value of approximately $6 \mathrm{~dB}$. The radiation pattern of the antenna assures a near hemispherical coverage toward the upper hemisphere.
\end{abstract}

Keywords-Axial ratio (AR), circular polarization (CP), Wideband Antennas, Wi-Max.

\section{INTRODUCTION}

Nowadays, the internet of things (IoT) gets high attention for both research and industrial communities. As a part of the internet of the future, IoT will constitute billions of intelligent autonomous communicating devices. Among several communicating protocols for an IoT device such as ZigBee or Wi-Fi, Wi-Max is considered as one of the most efficient option when the communication distance is long and/or the needed data rate is high. Considering that lots of IoT devices are dedicated to operate in an harsh environment, physical phenomenon such as polarization mismatch, multi-path interferences, and the Faraday Effect have a significant impact on the reliability of the IoT system [1]. In such situations, circularly polarized (CP) antennas are considered more efficient and a better choice comparing to linearly polarized (LP) ones.

As one the most interesting IoT applications, designing WLAN/Wi-Max antennas with a low profile will permit easier integration of these antennas. It is also a vital element when an embedded system is targeted where a lightweight of the IoT device is recommended. This is generally related to lowering the power consumption or controlling the device center of mass. However, reaching high performances in while employing a planar design could be a complex task to accomplish, especially when severe constraints are imposed. One of probable solutions is the employment of a threedimensional structures. Indeed, thanks to the third dimension, 3D-antennas exhibit better performance than 2Dones of the same size when properly designed [2]. It also gives a higher degree of freedom of the geometrical shape of the antenna, which makes it a promising and suitable solution for problems that present very conflicting requirements. Among many three dimensional antennas design, G. Hornby and J. Lohn presented in [3]-[5] a threedimensional wire antenna topology that has high performances in term of gain, space coverage, and polarization. It consists of six straight connected segments oriented arbitrary in space and operates above a ground plane. The use of a fully metallic wire antenna offers high radiation efficiency. Regarding the flexibility of this design and reliability of the measured results in the cited works, we envisioned to employ this topology.

IoT communication protocol defers from an application to another. In this work, we have chosen the WLAN (2.4$2.48 \mathrm{GHz}$ ) and Wi-Max band (3.3-3.7 GHz). The designed antenna covers the both WLAN and Wi-Max frequency bands and offers a CP for the Wi-Max band. One of the challenges is to maintain a proper matching with a $50 \Omega$ source. To design this $3 \mathrm{D}$ antenna, the use of computational optimization technique is mandatory because of the complex configurations. In the present case, a multi-objective genetic algorithm is employed. It is developed using MATLAB in conjunction with the electromagnetic simulator "Computer Simulation Technology Micro Wave Studio" (CST MWS).

This antenna could be used in many IoT applications and devices, such as IoT scenarios that involve drones or Unmanned ground and aerial vehicles. (UGV \& UAV).

\section{ANTENNA DESIGN AND RESULTS}

\section{A. Design Technique}

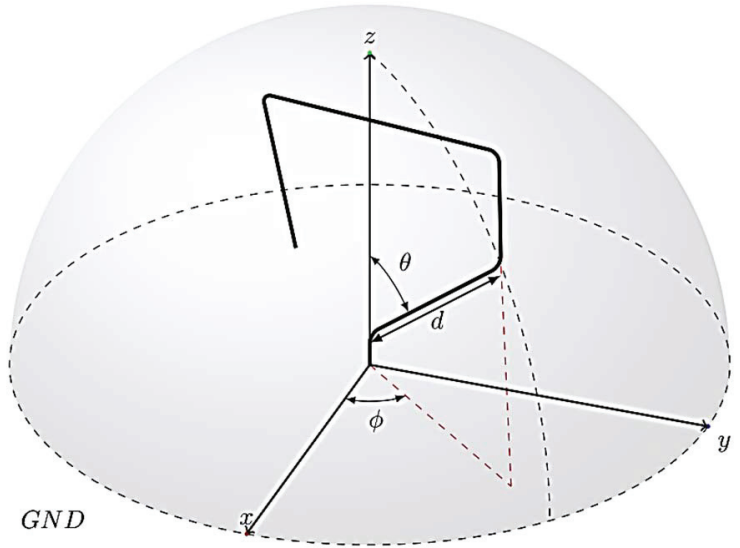

Fig. 1 Parametrization of the 3D antenna

The topology used in this work is a multi-segment wire monopole antenna. It consists of a fixed number of straight segments oriented randomly in space. The implemented multi-objective genetic approach starts by generating a number $N$ of individuals (vectors) that describe the antenna 
geometrical shape defined by the following parameters: $d_{i}$, the length of each segment, $\varphi_{i}$ and $\theta_{i}$, the azimuth and elevation angles respectively. The former and the later define the orientation of the segment in space. Figure 1 shows the parametrization of the active 3D antenna.

Besides the genetic-based optimization tool, a geometrical filter was developed and included in the optimization process in order to avoid wrong or unfeasible geometrical shapes, more specifically shapes with segments that go below the ground plane $(z=0)$ or with intersecting segments. It also excludes geometrical shapes with very sharp angles to respect fabrication constraints. Also, all structures that have a volume higher than $0.3 \lambda \times 0.3 \lambda \times 0.3 \lambda$ are excluded to limit the search inside acceptable volume of the final structure.

The implemented genetic algorithm employs the concepts of non-dominated sorting [6] which allows the use of multiple fitness functions without the need of weighted factors rather than a single one. Also, the crowding distance criterion [7] is implemented in order to conserve diversity in each generation and to avoid the convergence to very similar solutions. Table 1 resumes the fitness functions used.

TABLE 1

FITNESS FUNCTIONS

\begin{tabular}{lc}
\hline & Expression \\
\hline$f_{1}$ & $\left|S_{I I}\right|\left(@ \Delta f_{r l}\right)$ \\
\hline$f_{2}$ & $\left|S_{I I}\right|\left(@ \Delta f_{r 2}\right)$ \\
\hline$f_{3}$ & Axial Ratio $\left(@ \Delta f_{r 2}\right)$ \\
\hline \hline
\end{tabular}

In Table. $1, \Delta f_{r 1}$ and $\Delta f_{r 2}$ represent the frequency intervals (2.4-2.483) GHz and (3.3-3.7) GHz respectively. The axial ratio criterion was applied only on the Wi-Max frequency band. A frequency step of $10 \mathrm{MHz}$ is chosen to evaluate the fitness functions $f i$ while $100 \mathrm{MHz}$ is fixed for $f_{2}$.

The optimization tool evaluates all the individuals of a generation and selects the best according to the fitness functions and the crowding distance criterion. Those elements are transferred to the next generation. During transfer, cross-over and mutation are applied to the population with rates of $70 \%$ and $10 \%$ respectively. The number of individuals per generation is fixed to 120 .

The optimization tool is halted when no significant amelioration is recorded in any of three fitness functions. In the present study, it happened at the $637^{\text {th }}$ generation. Among the population of the last generation, one individual that shows good performances in term of adaptation $\left(S_{11}\right)$ and axial ratio is chosen. The structure is simulated another time in more realistic conditions, including the SMA connector and a limited ground plane. This can't be done during the optimization process because of the time that it would requires. Performances are not so different than the ones obtained previously.

\section{B. Fabrication of a Prototype}

The chosen structure was fabricated. For that, we used a $3 \mathrm{D}$-printer, the $3 \mathrm{ntr} \mathrm{A} 4 \mathrm{v} 3$, to fabricate a model of the antenna using a polymer adapted to Fused Deposition Modeling (FDM) 3D printing technique [8]. The printed structure was fixed to a SMA connector, and finally, we applied a thin layer of metallization on the external surface of the printed structure using conductive silver ink. The radius of the wire structure is $0.65 \mathrm{~mm}$, and it has a total length of $1.89 \lambda(237 \mathrm{~mm})$. The antenna fit into a box of $0.27 \lambda$ $\times 0.24 \lambda \times 0.29 \lambda$ which is equal to $33.8 \mathrm{~mm} \times 30.4 \mathrm{~mm} \times 37.4$ $\mathrm{mm}$. Fig. 2 shows the final fabricated prototype.

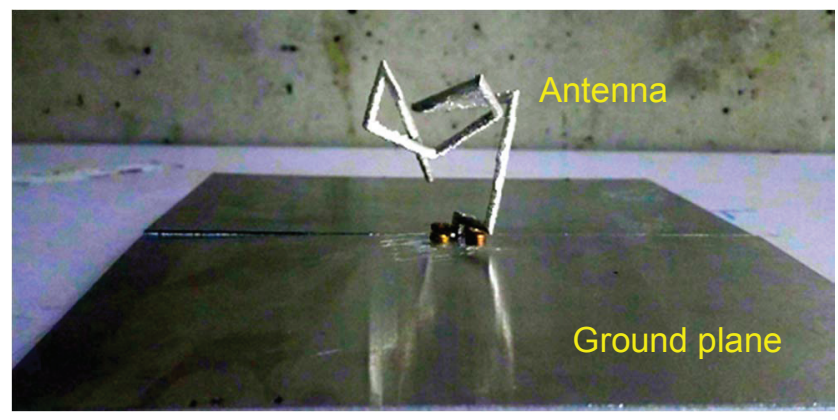

Fig. 2 Fabricated prototype.

\section{EXPERIMENTAL VALIDATION}

The fabricated prototype was tested to evaluate its reflection coefficient, $S_{11}$, its radiation pattern, and its polarization axial ratio. The measurements were done in an anechoic chamber using a vector network analyzer and two identical horn antennas, one as the emitting antenna and the second one for the calibration of the setup.

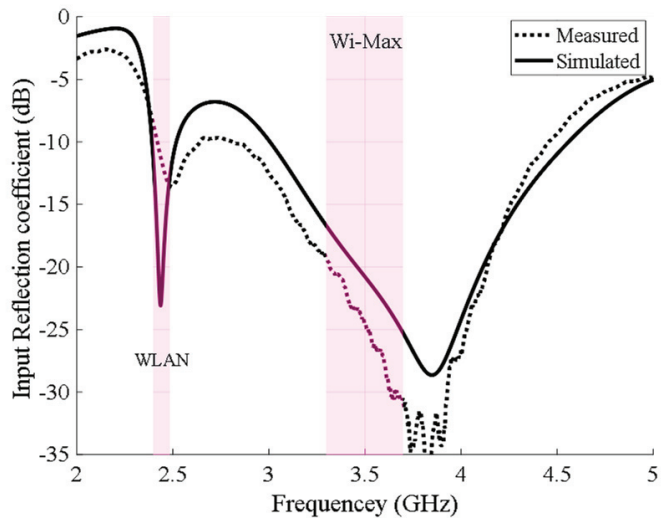

(a)

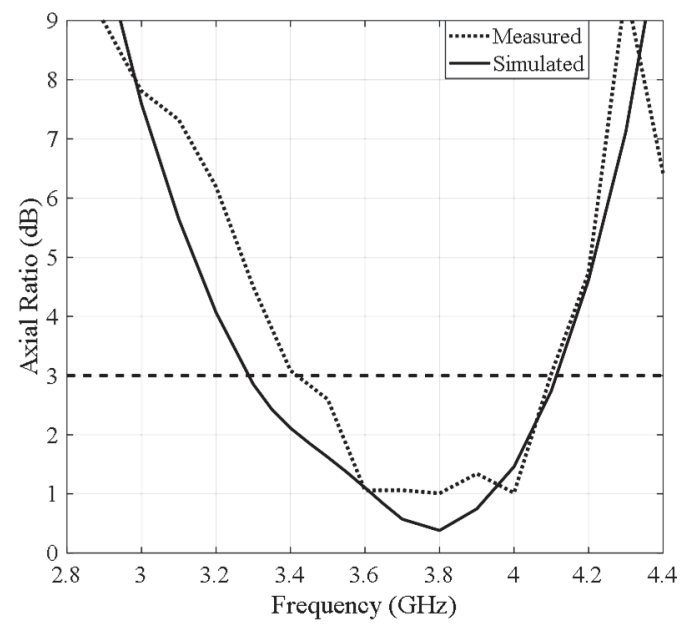

(b)

Fig. 3 Comparison between simulated and measured results. (a) Input reflection coefficient, $S_{11}$. (b) Axial ratio at the zenith. 
Fig. 3(a) shows the comparison between the simulation results using CST MWS and the measurements. We can notice that the measured reflection coefficient values of the fabricated prototype are close to the simulated results. As it was estimated, the fabricated prototype covers the frequency band (2.42-2.64) and (2.8-4.45) $\mathrm{GHz}$ with a reflection coefficient below $-10 \mathrm{~dB}$. This comprises the WLAN (2.4$2.483 \mathrm{GHz}$ ) and Wi-Max bands (3.3-3.7 GHz). A slightly degraded input reflection coefficient value is measured in the frequency $2.4-2.42 \mathrm{GHz}$ with values between -8.7 and -10 $\mathrm{dB}$. However, these values remain acceptable for most modern reception systems. The primary cause of this degradation is generally due to the geometrical fabrication errors and uncertainty related to experimental conditions. The AR at the zenith is compared in Figure 3(b). The simulated AR bandwidth for $3 \mathrm{~dB}$ is $830 \mathrm{MHz}(3.28-4.11$ $\mathrm{GHz}$ ), and the measured one is $690 \mathrm{MHz}(3.41-4.1 \mathrm{GHz})$.

Fig. 4(a) shows the measurements environment. In Fig. 4(b), the simulated and measured maximum gain in the $x z$ plane are illustrated. The measured $x z$ and $y z$ radiation patterns are very similar.

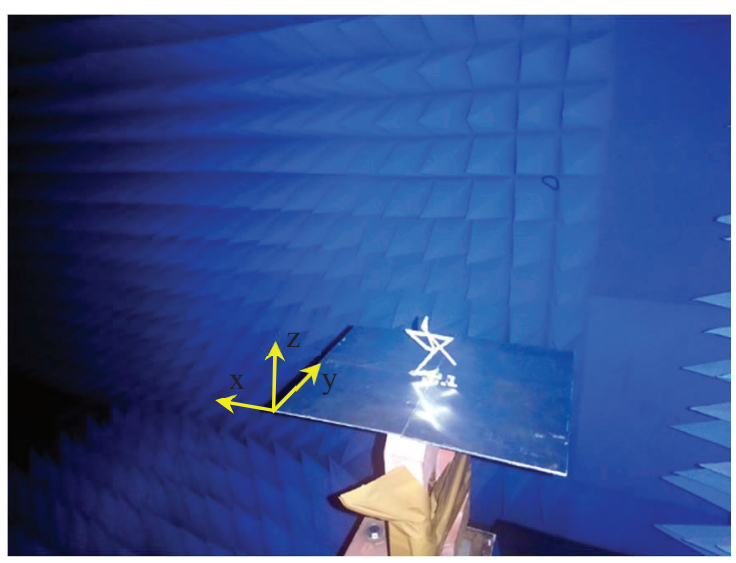

(a)

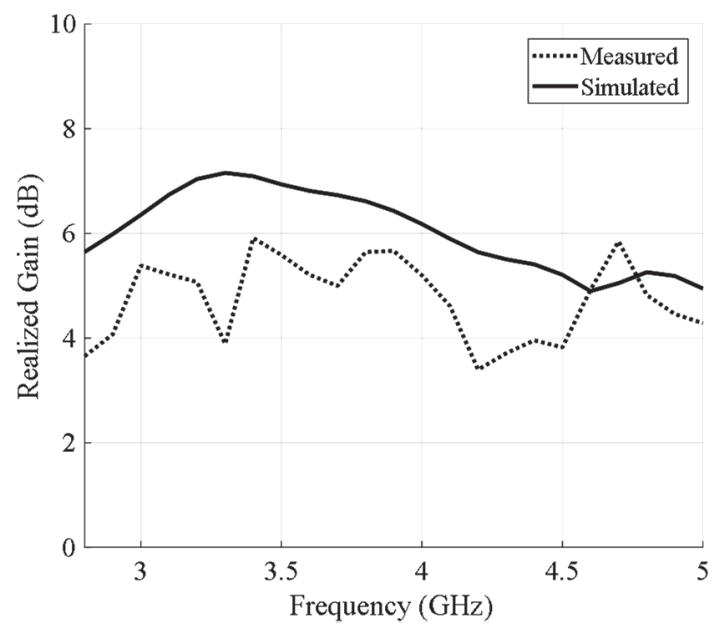

(b)

Fig. 4 Far-field measurements. (a) Measurements environment. (b) Simulated and measured gain in the $x z$ plane.

The simulated maximum gain is close to $7 \mathrm{~dB}$ in the $\mathrm{Wi}$ Max band. The measured gain was slightly lower with a value of approximately $5 \mathrm{~dB}$. The difference, $2 \mathrm{~dB}$, falls within the tolerances of the setup used. But more probably, it is also caused by the lower conductivity of the coating ink that affects the current flow on the radiating element leading to ohmic losses. The value of the realized gain remains high even when the adaptation is worst (above $4.5 \mathrm{GHz}$ ). This is explained by a high directivity near these frequencies. When the adaptation of the antenna moves away from $50 \Omega$, the antenna's directivity increases.

\section{CONCLUSION}

The design of a small size 3D-segmented monopole antenna is presented. It is double-band, circularly polarized It was realized and tested. It has a tridimensional geometry formed by ten straight segments arbitrary distributed in space. It is light, thus it makes it suitable for aerospatiale and drones' applications involved in IoT scenarios. Future works will focus on the test of alternative fabrication techniques.

\section{REFERENCES}

[1] C. C. Counselman, "Multipath-rejecting GPS antennas," Proc. IEEE, 1999.

[2] S. R. Best, "A Discussion on the Significance of Geometry in Determining the Resonant Behavior of Fractal and Other NonEuclidean Wire Antennas," in IEEE Antennas and Propagation Magazine, 2003.

[3] J. D. Lohn, G. S. Hornby, and D. S. Linden, "Human-competitive evolved antennas," Artif. Intell. Eng. Des. Anal. Manuf. AIEDAM, vol. 22, no. 3, pp. 235-247, 2008.

[4] G. S. Hornby, J. D. Lohn, and D. S. Linden, "ComputerAutomated evolution of an X-band antenna for NASA's space technology 5mission," Evol. Comput., vol. 19, no. 1, pp. 1-23, 2011

[5] J. D. Lohn, D. S. Linden, B. Blevins, T. Greenling, and M. R. Allard, "Automated synthesis of a lunar satellite antenna system," IEEE Trans. Antennas Propag., vol. 63, no. 4, pp. 1436-1444, 2015 .

[6] K. Deb, A. Pratap, S. Agarwal, and T. Meyarivan, "A fast and elitist multiobjective genetic algorithm: NSGA-II," IEEE Trans. Evol. Comput., 2002.

[7] F.-A. Fortin and M. Parizeau, "Revisiting the NSGA-II crowding-distance computation," 2013.

[8] S. H. Masood, "Advances in Fused Deposition Modeling," in Comprehensive Materials Processing, 2014. 